\title{
The use of meditative movement as a non-pharmacologic approach in hematological cancer patients: A systematic review of the literature
}

\author{
Ryan Eckert ${ }^{1 *}$, Jennifer Huberty ${ }^{2}$, Linda Larkey ${ }^{3}$, Lisa Marks ${ }^{4}$ and Ruben Mesa ${ }^{1}$ \\ ${ }^{1}$ Mays Cancer Center, UT Health San Antonio, San Antonio, TX 78229, USA \\ ${ }^{2}$ Exercise Science and Health Promotion, Arizona State University, Phoenix, AZ 85004, USA \\ ${ }^{3}$ College of Nursing and Health Innovation, Arizona State University, Phoenix, AZ 85004, USA \\ ${ }^{4}$ Division of Education, Library Services, Mayo Clinic, Scottsdale, AZ 85259, USA
}

\begin{abstract}
Introduction: Hematological cancer patients suffer from long-term physical and psychological comorbidities associated with treatment and disease course, including anxiety, depression, fatigue, and reduced quality of life. Meditative movement (i.e., yoga, qigong tai chi) is a form of complementary and integrative medicine that has received growing interest in recent decades. Meditative movement is efficacious for improving a variety of outcomes in other cancers, including mood, fatigue and quality of life. There is much less research examining the effects of meditative movement on hematological cancer patients. The purpose of this study was to systematically review the literature related to the effects of meditative movement specifically in hematological cancers. The findings of this review will help in making recommendations for future research.
\end{abstract}

Methods: Search strategies were developed by a medical librarian to scan the literature for published studies examining the effects of meditative movement (i.e., yoga, tai chi, or qigong) on hematological cancer patients.

Results: Four studies met our eligibility criteria.

Conclusions: There is preliminary evidence to support the effects of meditative movement on fatigue and sleep quality among hematological cancer patients, but particularly regarding yoga and qigong. No research has been done investigating tai chi on hematological cancer patients. We suggest the need for future research to continue to examine the effects of meditative movement on hematological cancer patients and to determine the minimal dose at which the effects of meditative movement begin to occur.

\section{Introduction}

Hematological cancers are a group of blood- and lymph-related disorders, of which the most common sub-types include leukemia, lymphoma (i.e., Hodgkin and non-Hodgkin), and myeloma [1]. Although less prevalent than some of the more common cancer types (i.e., breast, prostate, lung, colorectal), hematological cancers accounted for $\sim 10 \%$ of all new cancer diagnoses and cancer deaths in 2017 [2]. Symptom profiles vary by hematological cancer sub-type, with certain symptoms similar to those experienced by other types of cancer patients (i.e., fatigue, weight loss, pain) and others being somewhat unique to hematological cancers (i.e., anemia, pruritis, bleeding or bruising easily) $[3,4]$. In general, hematological treatments include chemotherapy and/or other drug therapy, radiation, immunotherapy, blood transfusions, or stem-cell transplantation [1].

The five-year relative survival rate of hematological cancer patients in the United States has seen a significant increase over the past half century. Among the most common hematological cancer sub-types (i.e., leukemia, lymphoma, myeloma) five-year relative survival rates have increased from between $12-40 \%$ to between $50-89 \%$ depending on diagnosis [2]. Although promising, this also means that there is an increased need to provide long-term care to both hematological cancer patients currently undergoing treatment as well as survivors. It has been shown that hematological cancer patients and survivors often suffer from psychological comorbidities, including anxiety, depressive symptoms, and fatigue [5-7]. Furthermore, each of the aforementioned comorbidities have been found to interfere with quality of life (QoL) and activities of daily living [5].

There has been a growing interest in and use of complementary and integrative medicine (CIM) among cancer patients, with the prevalence of CIM use in cancer patients increasing over the past 30 years from $\sim 25 \%$ to $\sim 50 \%$ of patients [8]. Additionally, cancer patients have been reported to be more likely to use CIM when compared to non-cancer populations [9]. Meditative movement is defined as practices that include some sort of postural movement, a focus on the breath, and incorporation of a meditative state to achieve deep states of relaxation (i.e., yoga, tai chi, qigong) [10]. Meditative movements are some of most commonly practiced CIM approaches, with $\sim 6.5 \%$ of cancer survivors in the U.S. reporting that they practice a form of meditative movement [11]. Additionally, meditative movement has been studied as a means of addressing some of the long-term side effects of cancer and its associated treatment. Given that meditative movement practices tend to have more in common (i.e., promoting similar body, breath

${ }^{\star}$ Correspondence to: Ryan Eckert, Mays Cancer Center, UT Health San Antonio, 7979 Wurzbach Road, San Antonio, TX 78229, USA, Tel: 781-588-0237; E-mail: eckertr@uthscsa.edu

Key words: mind-body, complementary, quality of life, cancer

Received: October 03, 2018; Accepted: October 15, 2018; Published: October 18,2018 
and mind states) than differences, albeit different movement types and intensities within and across modalities, we are treating these as a category for exploring effects on symptoms of hematological cancer patients and survivors.

Multiple systematic reviews and meta-analyses have found that meditative movement practices are efficacious for improving a range of outcomes in cancer patients. Yoga has been shown to improve functional well-being, distress, anxiety, depression, fatigue, emotional function, social function, sleep quality, and QoL in cancer patients [12-15]. Furthermore, Tai chi and qigong have evidence to support improvements in fatigue, mood, QoL, immune function, cortisol levels, and inflammation in cancer patients $[16,17]$. Much of the aforementioned work, however, has been conducted in solid tumor cancer patients (e.g., breast, liver, lung, prostate, etc.). There has been far less research examining the benefits of meditative movement on hematological cancer patients despite the benefits of this type of complementary treatment for other cancer types.

A review summarizing the effects of meditative movement in hematological cancer patients is needed. Therefore, the purpose of this study was to systematically review the literature on the effects of meditative movement in hematological patients and to provide direction for future research. To examine the effects of meditative movement on hematological cancer patient outcomes, we reviewed published randomized controlled trials and/or single-arm experimental trials that assessed the effects of yoga, tai chi, or qigong interventions specifically in hematological cancer sub-types. The findings of this review will help in 1) advancing our knowledge of the effects of various forms of meditative movement in hematological cancer patients and 2) making recommendations for future research.

\section{Methods}

\section{Search Strategy}

A medical librarian (LM) developed search strategies for Ovid MEDLINE, Ovid Embase, and PubMed to identify articles. The search strategies were peer-reviewed by another experienced medical librarian. Search strategies included the MeSH terms and key words in Table 1.

\section{Inclusion/Exclusion Criteria}

Studies were included if they: (a) were published between 1995 and 2018, (b) were published in English, (c) examined the effects of meditative movement (to include yoga, qigong, or tai chi [10] on patient outcomes (d) had an experimental (i.e., randomized controlled trial $[\mathrm{RCT}]$ ), quasi-experimental (i.e., single-arm interventions) or epidemiological (e.g., longitudinal, cross-sectional) study design, and

Table 1. Keyword Search Terms

\begin{tabular}{|l|l|}
\hline MeSH Terms (PubMed) & Keywords (PubMed) \\
\hline yoga & yoga \\
\hline Qigong & Qigong \\
\hline Tai Ji & Tai Chi \\
\hline Mind-Body Therapies & Mindful movement \\
\hline & Meditative movement \\
\hline & MBSR \\
\hline & Mindfulness based stress reduction \\
\hline hematologic neoplasms & Blood cancers \\
\hline multiple myeloma & Multiple myeloma \\
\hline leukemia & leukemia \\
\hline lymphoma & Lymphoma \\
\hline myeloproliferative disorders & Myeloproliferative disorders \\
\hline
\end{tabular}

(e) were conducted in specific hematological cancer sub-types (i.e., leukemia, lymphoma, myeloma, and/or myeloproliferative neoplasm patients/survivors). Systematic reviews, meta-analyses, studies that examined meditative movement in relation to hematological cancer risk, and conference abstracts were excluded.

\section{Selection Process}

Titles and abstracts of articles that were found in the search results were reviewed to exclude articles that were not relevant. Potentially relevant full-text articles were then scanned for eligibility by one reviewer (RE) and were then confirmed and agreed upon by a second reviewer $(\mathrm{JH})$. Only articles that met all inclusion criteria and were agreed upon to be included by both reviewers (RE \& JH) were included in this review. The references/bibliographies of included articles were also scanned for additional relevant articles that could be included in the review.

\section{Data Extraction}

The following data were extracted: (a) study design, (b) study population/sample, (c) type of meditative movement, (d) prescription (i.e., intervention duration, intensity, length of each session, type/ style of meditative movement), (e) effects of meditative movement on patient-reported outcomes, and (f) study limitations.

\section{Quality Assessment}

Quality assessment of individual studies was conducted for all studies included in the systematic review. Quality assessment was conducted using the 27-item checklist by Downs and Black [18], which can be used to assess both randomized and non-randomized trials. In the present version of the checklist, the checklist scoring was modified for item 27 that refers to the power of the study. Instead of rating according to an available range of study powers, it was rated by whether the study performed a power calculation or not. Accordingly, the maximum score for item 27 was one (a power analysis was conducted) instead of five and thus the highest possible score for the checklist was 28 (instead of 32). Downs and Black score ranges were given corresponding quality levels as previously reported: excellent (26-28); good (20-25); fair (15-19); and poor ( $\leq 14)$ [19]. Two individuals (RE \& LM) performed the quality assessment independently. Each assessor then met, reviewed their quality assessment scores, and resolved any discrepancies until quality assessment scores for each item were agreed upon.

\section{Results}

A total of 256 articles were found with the literature search conducted on July 23,2018 . A total of 90 duplicates were removed, leaving 166 articles that were screened for eligibility. A total of 151 were excluded after examining the titles of each as they did not meet our predefined inclusion criteria, leaving 15 potentially relevant articles left for review. After examining the full-texts of each of the 15 articles, four articles met our inclusion criteria and were included for review. Table 2 describes the study selection process and reasons for excluding articles (Figure 1). A detailed description of the included articles is included in the next section below (Table 2).

\section{Quality Assessment}

Results of the quality assessment are presented in Table 3. The four studies included were rated as "good" [20,21] and "fair" [22,23] quality according to the Downs and Black [18] checklist (Table 3). 
Eckert R (2018) The use of meditative movement as a non-pharmacologic approach in hematological cancer patients: A systematic review of the literature

Table 2. Summary of Included Articles

\begin{tabular}{|c|c|c|c|c|c|c|}
\hline Reference & Study Design & Study Sample & $\begin{array}{l}\text { Type of Meditative } \\
\text { Movement }\end{array}$ & Prescription & $\begin{array}{l}\text { Results \& Significant } \\
\text { Findings }\end{array}$ & Limitations \\
\hline Cohen, et al. [21] & $\begin{array}{l}\text { RCT (wait-list control } \\
\text { group) }\end{array}$ & $\begin{array}{l}\text { Lymphoma patients } \\
(\mathrm{n}=38 ; 19 \text { in } \\
\text { intervention })\end{array}$ & Yoga & $\begin{array}{l}7 \text { weeks; } 1 \mathrm{x} / \text { week; } \\
\text { light-intensity; unknown } \\
\text { session duration; Tibetan } \\
\text { yoga }\end{array}$ & $\begin{array}{l}89 \% \text { completed } 2-3 \\
\text { sessions; } 57 \% \text { completed } \\
\geq 5 \text { sessions; } \downarrow \text { sleep } \\
\text { disturbance; } \uparrow \text { sleep } \\
\text { quality; faster sleep } \\
\text { latency; } \downarrow \text { use of sleep } \\
\text { meds }\end{array}$ & $\begin{array}{l}\text { Low overall exercise } \\
\text { dose; small sample size } \\
\text { in intervention group } \\
(\mathrm{n}=20) \text {; yoga session } \\
\text { duration not specified }\end{array}$ \\
\hline Huberty et al. [23] & $\begin{array}{l}\text { Quasi-Experimental } \\
\text { (single-arm feasibility } \\
\text { design) }\end{array}$ & $\begin{array}{l}\text { Myeloproliferative } \\
\text { neoplasm patients }(n=38)\end{array}$ & Yoga & $\begin{array}{l}12 \text { weeks } ; 00 \mathrm{~min} / \text { week; } \\
\text { mild/moderate-intensity; } \\
20-30 \text { min sessions; style } \\
\text { of yoga varied }\end{array}$ & $\begin{array}{l}\text { Average yoga } \\
\text { participation of } \sim 50 \mathrm{~min} / \\
\text { week; } 37 \% \text { averaged } \\
\geq 60 \mathrm{~min} / \text { week; } \downarrow \text { total } \\
\text { symptom burden; } \\
\downarrow \text { fatigue; } \downarrow \text { anxiety; } \\
\downarrow \text { depression; } \downarrow \text { sleep } \\
\text { disturbance }\end{array}$ & $\begin{array}{l}\text { Small sample size } \\
(\mathrm{n}=38) \text {; no control group } \\
\text { to test for effectiveness; } \\
\text { potential bias due to } \\
\text { study being advertised as } \\
\text { yoga intervention; biased } \\
\text { proportion of females } \\
\text { in study }\end{array}$ \\
\hline Yeh, et al. [24] & $\begin{array}{l}\text { RCT (usual care control } \\
\text { group) }\end{array}$ & $\begin{array}{l}\text { Non-Hodgkin's } \\
\text { lymphoma patients } \\
(\mathrm{n}=102 ; 51 \text { in } \\
\text { intervention) }\end{array}$ & Qigong & $\begin{array}{l}21 \text { days; } 20-60 \mathrm{~min} / \\
\text { session (including a } \\
5 \text {-min warm-up); } 2-5 \mathrm{x} \\
\text { daily; Chan-Chuang } \\
\text { qigong }\end{array}$ & $\begin{array}{l}\text { Qigong participation } \\
\text { averaged } \sim 47 \mathrm{~min} / \text { day; } \\
\downarrow \text { average fatigue; } \downarrow \text { worst } \\
\text { fatigue; } \uparrow \text { overall sleep } \\
\text { quality }\end{array}$ & $\begin{array}{l}\text { Significant differences at } \\
\text { baseline between groups } \\
\text { for primary outcome } \\
\text { measures; short duration } \\
\text { of study (i.e., } 21 \text { days); } \\
\text { lack of placebo control } \\
\text { group; self-report qigong } \\
\text { participation }\end{array}$ \\
\hline Chuang, et al. [22] & $\begin{array}{l}\text { RCT (usual care control } \\
\text { group) }\end{array}$ & $\begin{array}{l}\text { Non-Hodgkin's } \\
\text { lymphoma patients } \\
(\mathrm{n}=96 ; 48 \text { in } \\
\text { intervention })\end{array}$ & Qigong & $\begin{array}{l}21 \text { days; } 25 \mathrm{~min} / \mathrm{session} \\
\text { (including a } 5-\mathrm{min} \\
\text { warm-up and 5-min cool- } \\
\text { down); } 2-3 \mathrm{x} / \text { day; Chan- } \\
\text { Chuang qigong }\end{array}$ & $\begin{array}{l}\text { A total of } 96 \% \text { of } \\
\text { patients practiced qigong } \\
\text { on } 21 \text { days and average } \\
\text { daily qigong participation } \\
\text { averaged } ~ 48 \text { min/day; } \\
\downarrow \text { fatigue intensity \& } \\
\text { interference; } \uparrow W B C \text { count, } \\
\text { hemoglobin, and platelets; } \\
\uparrow \text { sleep quality; } \uparrow Q \text { QL }\end{array}$ & $\begin{array}{l}\text { significant differences at } \\
\text { baseline between groups } \\
\text { in fatigue, sleep quality, } \\
\text { and QoL sub-domains; } \\
\text { short duration of study } \\
\text { (i.e., } 21 \text { days) }\end{array}$ \\
\hline
\end{tabular}

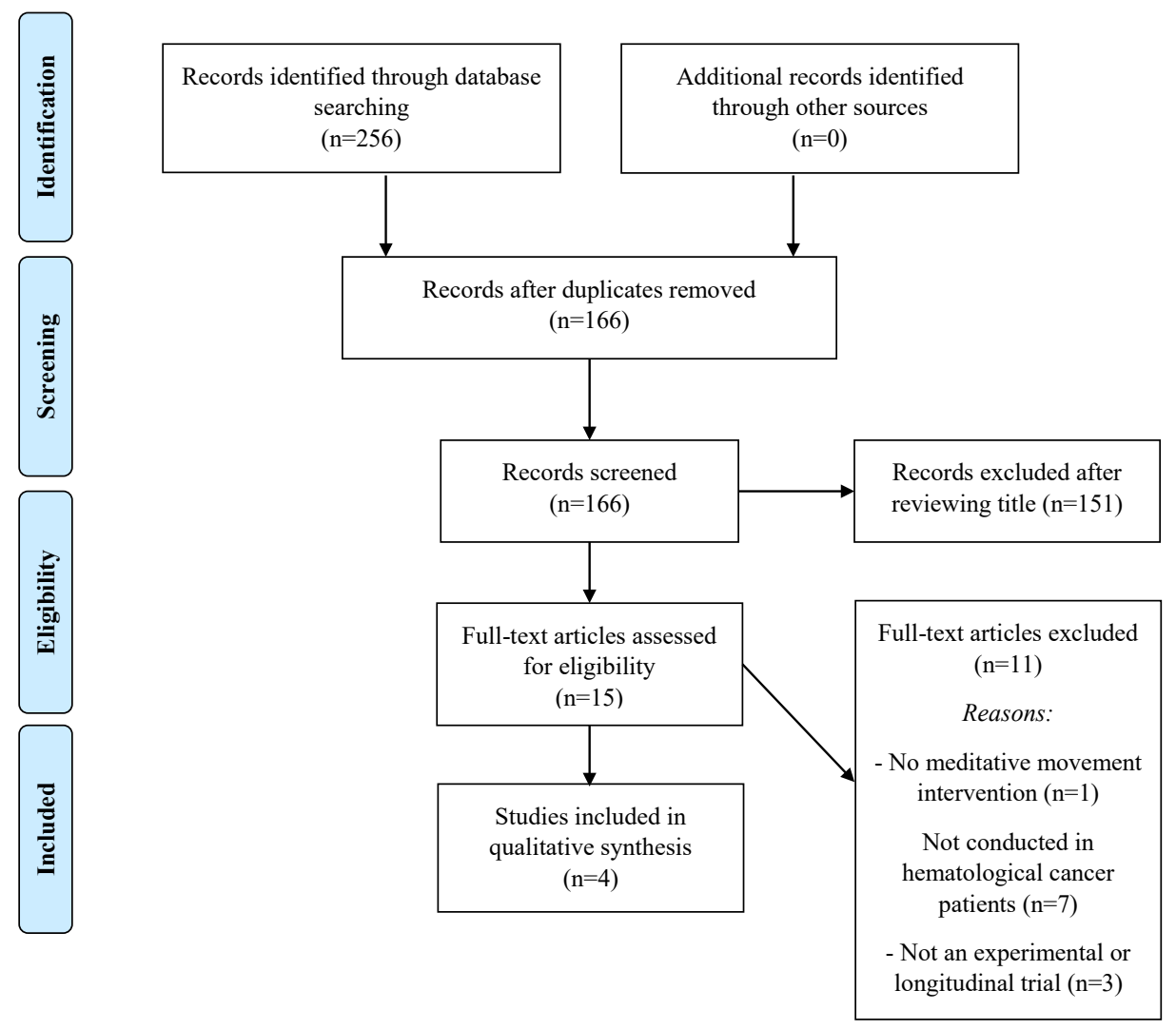

Figure 1. Study Selection Flow Chart 
Table 3. Downs and Black Quality Assessment Checklist

\begin{tabular}{|c|c|c|c|c|c|}
\hline \multirow[b]{2}{*}{ Item } & \multirow[b]{2}{*}{ Scale } & \multicolumn{4}{|c|}{ Study Reference } \\
\hline & & $\begin{array}{l}\text { Cohen, et } \\
\text { al. }[121]\end{array}$ & $\begin{array}{l}\text { Huberty, } \\
\text { et al. }[23]\end{array}$ & $\begin{array}{l}\text { Yeh, et } \\
\text { al. }[24]\end{array}$ & $\begin{array}{l}\text { Chuang et } \\
\text { al.[22] }\end{array}$ \\
\hline \multicolumn{6}{|l|}{ Reporting } \\
\hline 1. Is the hypothesis/aim/objective of the study clearly described? & $\mathrm{Yes}=1 ; \mathrm{no}=0$ & yes & yes & yes & yes \\
\hline $\begin{array}{l}\text { 2. Are the main outcomes to be measured clearly described in the Introduction or } \\
\text { Methods section? }\end{array}$ & Yes $=1 ;$ no $=0$ & yes & yes & yes & yes \\
\hline 3. Are the characteristics of the patients included in the study clearly described? & Yes $=1 ;$ no $=0$ & yes & yes & yes & yes \\
\hline 4. Are the interventions of interest clearly described? & Yes $=1 ;$ no $=0$ & yes & yes & yes & yes \\
\hline $\begin{array}{l}\text { 5. Are the distributions of principal confounders in each group of subjects to be } \\
\text { compared clearly described? }\end{array}$ & Yes $=2 ;$ partially $=1 ;$ no $=0$ & no & no & no & no \\
\hline 6. Are the main findings of the study clearly described? & $\mathrm{Yes}=1 ; \mathrm{no}=0$ & yes & yes & yes & yes \\
\hline $\begin{array}{l}\text { 7. Does the study provide estimates of the random variability in the data or the main } \\
\text { outcomes? }\end{array}$ & Yes $=1 ;$ no $=0$ & yes & yes & yes & yes \\
\hline $\begin{array}{l}\text { 8. Have all important adverse events that may be a consequence of the intervention been } \\
\text { reported? }\end{array}$ & Yes $=1 ;$ no $=0$ & no & yes & yes & yes \\
\hline 9. Have the characteristics of patients lost to follow-up been described? & Yes $=1 ;$ no $=0$ & yes & yes & no & yes \\
\hline $\begin{array}{l}\text { 10. Have actual probability values been reported (e.g. } 0.035 \text { rather than }<0.05 \text { ) for the } \\
\text { main outcomes except where the probability value is less than } 0.001 \text { ? }\end{array}$ & Yes $=1 ; n o=0$ & yes & yes & yes & yes \\
\hline \multicolumn{6}{|l|}{ External Validity } \\
\hline $\begin{array}{l}\text { 11. Were the subjects asked to participate in the study representative of the entire } \\
\text { population from which they were recruited? }\end{array}$ & Yes $=1 ;$ no $=0 ;$ unable to determine $=0$ & yes & yes & yes & yes \\
\hline $\begin{array}{l}\text { 12. Were those subjects who were prepared to participate representative of the entire } \\
\text { population from which they were recruited? }\end{array}$ & Yes $=1 ;$ no $=0$; unable to determine $=0$ & yes & yes & yes & yes \\
\hline $\begin{array}{l}\text { 13. Were the staff, places, and facilities where the patients were treated, representative of } \\
\text { the treatment the majority of patients receive? }\end{array}$ & Yes $=1 ;$ no $=0 ;$ unable to determine $=0$ & yes & yes & yes & yes \\
\hline \multicolumn{6}{|l|}{ Internal Validity - Bias } \\
\hline 14. Was an attempt made to blind study subjects to the interventionthey have received? & Yes $=1 ;$ no $=0 ;$ unable to determine $=0$ & $\begin{array}{l}\text { Unable to } \\
\text { determine }\end{array}$ & no & no & no \\
\hline $\begin{array}{l}\text { 15. Was an attempt made to blind those measuring the main outcomes of the } \\
\text { intervention? }\end{array}$ & Yes $=1 ;$ no $=0 ;$ unable to determine $=0$ & $\begin{array}{l}\text { Unable to } \\
\text { determine }\end{array}$ & $\begin{array}{l}\text { Unable to } \\
\text { determine }\end{array}$ & no & yes \\
\hline 16. If any of the results of the study were based on "data dredging", was this made clear? & Yes $=1 ;$ no $=0 ;$ unable to determine $=0$ & yes & yes & yes & yes \\
\hline $\begin{array}{l}\text { 17. In trials and cohort studies, do the analyses adjust for different lengths of follow-up } \\
\text { of patients, or in case-control studies, is the time period between the intervention and } \\
\text { outcome the same for cases and controls? }\end{array}$ & Yes $=1 ;$ no $=0 ;$ unable to determine $=0$ & yes & yes & no & yes \\
\hline 18. Were the statistical tests used to assess the main outcomes appropriate? & Yes $=1 ;$ no $=0$; unable to determine $=0$ & yes & yes & yes & yes \\
\hline 19. Was compliance with the intervention/s reliable? & Yes $=1 ;$ no $=0 ;$ unable to determine $=0$ & no & no & no & no \\
\hline 20. Were the main outcome measures used accurate (valid and reliable)? & Yes $=1 ;$ no $=0 ;$ unable to determine $=0$ & yes & yes & yes & yes \\
\hline \multicolumn{6}{|l|}{ Internal Validity - Confounding (Selection Bias) } \\
\hline $\begin{array}{l}\text { 21. Were the patients in different intervention groups (trials and cohort studies) or were } \\
\text { the cases and controls (case-control studies) recruited from the same population? }\end{array}$ & Yes $=1 ;$ no $=0 ;$ unable to determine $=0$ & yes & yes & yes & yes \\
\hline $\begin{array}{l}\text { 22. Were study subjects in different intervention groups (trials and cohort studies) or } \\
\text { were the cases and controls (case-control studies) recruited over the same period of time? }\end{array}$ & Yes $=1 ;$ no $=0 ;$ unable to determine $=0$ & yes & no & yes & yes \\
\hline 23. Were study subjects randomized to intervention groups? & Yes $=1 ;$ no $=0 ;$ unable to determine $=0$ & yes & no & yes & yes \\
\hline $\begin{array}{l}\text { 24. Was the randomised intervention assignment concealed from both patients and health } \\
\text { care staff until recruitment was complete and irrevocable? }\end{array}$ & Yes $=1 ;$ no $=0 ;$ unable to determine $=0$ & yes & no & no & no \\
\hline $\begin{array}{l}\text { 25. Was there adequate adjustment for confounding in the analyses from which the main } \\
\text { findings were drawn? }\end{array}$ & Yes $=1 ;$ no $=0 ;$ unable to determine $=0$ & no & no & no & no \\
\hline 26. Were losses of patients to follow-up taken into account? & Yes $=1 ;$ no $=0$; unable to determine $=0$ & $\begin{array}{l}\text { Unable to } \\
\text { determine }\end{array}$ & yes & yes & yes \\
\hline \multicolumn{6}{|l|}{ Power } \\
\hline \multirow[t]{2}{*}{ 27. Did the study conduct a power calculation? } & Yes $=1 ;$ no $=0 ;$ unable to determine $=0$ & yes & no & yes & yes \\
\hline & Total Score (total possible score of 28 ) & 20 (good) & 18 (fair) & 19 (fair) & 22 (good) \\
\hline
\end{tabular}

\section{Effects of Meditative Movement in Hematological Cancers}

A study conducted in 2004 by Cohen, et al. [21] examined the effects of yoga on lymphoma patients. Patients $(n=39)$ were randomized to participate in a yoga intervention group $(n=20)$ or a wait-list control group $(n=19)$. Only one participant dropped out in the yoga intervention group, leaving a total of 38 patients that completed the study ( $\mathrm{n}=19$ in each group). The intervention group performed inperson Tibetan yoga (e.g., breathing, visualization, mindfulness, and low-impact postures) at the University of Texas MD Anderson Cancer Center $1 \mathrm{x} /$ week for seven weeks (duration of each session was not specified). Each yoga session was led by an experienced Tibetan Yoga instructor. The primary outcomes for this study included sleep quality measures (i.e., overall quality, latency, duration, sleep medication use), state anxiety, depression, and fatigue. Although significant improvements in subjective sleep quality, shorter sleep latency, longer sleep duration, and less reported use of sleep medications were found, there were no significant differences between groups for state anxiety, depression or fatigue. However, this may be due, in part, to both the adherence rate as only $58 \%$ of patients in the intervention group completed at least five of the seven prescribed yoga sessions. 
A study conducted by Yeh, et al. [20] aimed to evaluate the effects of qigong on non-Hodgkin's lymphoma patients undergoing chemotherapy. This RCT randomly assigned 108 non-Hodgkin's lymphoma patients to either a qigong intervention group or a usual care control group. The qigong group was asked to participate in selfdirected qigong (i.e., on their own with the help of an instruction manual) for 21 consecutive days, 2-5x/day, and 20-60 min/session during the course of chemotherapy treatment. Qigong participants were provided with a modified version of a Chan-Chuang qigong instruction manual and were taught on a single day how to practice qigong by a research nurse who was trained by two qualified qigong practitioners. The control group received their normal course of chemotherapy treatment and was not asked to participate in qigong. The primary outcomes for this study included fatigue intensity (i.e., average fatigue, worst fatigue) and sleep quality measures (i.e., sleep disturbance, sleep effectiveness, sleep supplementation, overall sleep quality) and were assessed every seven days throughout the study (i.e., day $0,7,14, \& 21)$.

A total of 108 patients signed an informed consent. Of these 108 patients, 54 were randomized to each group. Six patients dropped out, leaving a total of 102 patients ( $\mathrm{n}=51$ in each group) that completed the study. Overall qigong participation averaged $\sim 47 \mathrm{~min} /$ day for the 21-day study duration. There were significant improvements over time in the qigong group compared to the control group for average fatigue $(\mathrm{p}<0.001)$, fatigue intensity $(\mathrm{p}<0.001)$ and overall sleep quality $(\mathrm{p}<0.001)$. There are several limitations of this study, including 1$)$ the short study duration (i.e., 21 days), 2) the lack of placebo control group, 3) self-report qigong participation, and 4) significant differences between the intervention and control groups at baseline for primary outcomes (i.e., fatigue intensity and sleep quality).

Another study conducted by Chuang, et al. [22] evaluated the effectiveness of qigong for fatigue, complete blood counts, sleep quality, and QoL in non-Hodgkin's lymphoma patients undergoing chemotherapy. This RCT randomly assigned 100 non-Hodgkin's lymphoma patients to either a qigong intervention group or a usual care control group. The methods for this study were similar to those of the previous study by Yeh, et al. [20,22] and asked participants to complete 25-min qigong sessions on their own 2-5x/day for 21 consecutive days after receiving chemotherapy treatment. The primary outcomes for this study included fatigue intensity, fatigue interference, complete blood cells (i.e., white blood cells, hemoglobin, platelets) sleep quality measures (i.e., sleep disturbance, sleep effectiveness, sleep supplementation, overall sleep quality) and QoL and were assessed every seven days throughout the study (i.e., day $0,7,14, \& 21$ ).

A total of 100 patients signed an informed consent. Of these 100 patients, 50 were randomized to each group. Four patients dropped out, leaving 96 patients ( $\mathrm{n}=48$ in each group) that completed the study. A total of $96 \%$ of patients $(n=46 / 48)$ practiced qigong on 21 days and daily qigong participation across all participants averaged $\sim 48 \mathrm{~min} /$ day. There was a significant decrease over time for the qigong group compared to the control group for fatigue intensity and fatigue interference. Additionally, there were significant increases over time in the qigong group compared to the control group for white blood cells, hemoglobin, and platelets as well as sleep quality and QoL. Limitations to note for this study include 1) significant differences at baseline between the groups for fatigue measures, sleep quality, and QoL and 2) the short duration of the study (i.e., 21 days).

A recent study conducted by Huberty, et al. [23] aimed to examine the feasibility and preliminary effectiveness of online-streamed yoga
(www.Udaya.com) for myeloproliferative neoplasm (MPN) patients. This was a single-arm feasibility trial that enrolled 38 patients to participate in $60 \mathrm{~min} /$ week of home-based, online-streamed yoga for 12 weeks. A 12 -week yoga prescription was provided to participants via Udaya.com, which is a consumer-based online-streaming yoga platform. Participants were asked to participate in a minimum of 60 $\mathrm{min} /$ week by completing 20-30 min videos, $2-3 \mathrm{x} /$ week. The type of yoga sessions included in the 12 -week prescription varied slightly but included primarily hatha-style yoga and meditation/mindfulness classes. Each class was led by an experienced, certified yoga instructor. The primary outcome was feasibility (i.e., practicality, acceptability and demand) and secondary outcomes included total symptom burden, fatigue, anxiety, depression, pain intensity, sexual function, and sleep disturbance. Participants completed questionnaires assessing demographics, symptom burden and study satisfaction at baseline, mid-point (week 7, post-intervention (week 12) and follow-up (week 16).

Yoga participation averaged $\sim 50 \mathrm{~min} /$ week and $37 \%$ of participants averaged $\geq 60 \mathrm{~min} /$ week of yoga (i.e., demand). A total of $68 \%(\mathrm{n}=21 / 28)$ of responding participants felt either satisfied or very satisfied with online yoga, $75 \%(n=23 / 31)$ felt that it was helpful for coping with MPN-related symptoms, and $75 \%$ of participants $(n=23 / 31)$ reported that they felt safe while participating in online yoga (acceptability). A total of $82 \%(n=31 / 38)$ completed the mid-point questionnaire, $79 \%(n=30 / 38)$ completed the post-intervention questionnaire, and $74 \%(n=28 / 38)$ completed the follow-up questionnaire. There were significant pre-post differences in total symptom burden $(\mathrm{p}=0.04$; $E S=-0.36)$, fatigue $(p=0.04 ; E S=-0.33)$, anxiety $(p=0.002 ; E S=-0.67)$, depression ( $\mathrm{p}=0.049 ; \mathrm{ES}=-0.41)$, and sleep disturbance $(\mathrm{p}<0.0001$; $\mathrm{ES}=-0.58$ ). There were no significant differences in outcomes between those that averaged $<60 \mathrm{~min} /$ week of yoga and those that averaged $>60$ $\mathrm{min} /$ week of yoga. Although this study was deemed to be feasible and demonstrated significant findings, limitations of this study include 1) the potential for selection bias due to the study being advertised as an online yoga study and 2) the lack of a control group and a small sample size, limiting the ability of the study to determine effectiveness.

\section{Discussion}

Research investigating the effects of meditative movement (i.e., yoga, tai chi, qigong) on hematological cancer patients is still in its infancy. Our study highlights the limited amount of research that has been done in the study of meditative movement for hematological cancer patients and the need for future interventions. Based on the findings of this review, there is some preliminary evidence to suggest that meditative movement may improve patient outcomes in hematological cancer patients, particularly fatigue and sleep quality as these outcomes were improved by meditative movement across the majority of studies $(n=3 / 4)$ included in our review.

Only four studies were included in our review, each of which carries its own limitations. Two studies included small sample sizes (i.e., $<40$ participants in both trials) [21,23] demonstrating the need for larger sample sizes in future studies. Additionally, the study by Cohen, et al. [21] included a low yoga dose that may partially explain a lack of significant effects of yoga on anxiety, depression, and fatigue. Seven prescribed sessions may not have been a potent enough dose to improve psychosocial outcomes (i.e., anxiety, depression, and fatigue). Previous yoga studies in breast cancer patients have demonstrated significant improvements in anxiety, depression, and fatigue after higher doses of yoga (i.e., 18-24, 60-minute sessions) [24-26] Furthermore, the study by Huberty, et al. [23] was a feasibility study that was not powered 
for effects and a study sample that was primarily female $(89.5 \%)$, potentially introducing bias and limiting generalizability.

We found two studies investigating yoga and two studies investigating qigong, whereas we did not find any studies that examined the effects of tai chi on hematological cancer patients. Future studies are warranted that examine the effects of tai chi on hematological cancer patients as tai chi has demonstrated to be beneficial in solid tumor cancer patients [27]. Additionally, further research is needed to better understand the effects of qigong and yoga on hematological cancer patients. There has been a growing body of literature surrounding the effects of yoga, qigong, and tai chi on other cancer patients with little literature in hematological cancers. Due to unique differences in etiology and treatment between solid tumor cancers and hematological cancers, research is needed that examines meditative movement in hematological cancers specifically.

Future research should aim to 1) examine the effects of meditative movement (i.e., yoga, tai chi, qigong) on hematological cancer patient outcomes (i.e., symptoms and QoL) and 2) determine the ideal dose of meditative movement for changes in symptoms and QoL. In the study conducted by Huberty, et al. [23] there were no significant differences in outcomes (i.e., total symptom burden, fatigue, anxiety, depression, sleep disturbance) between those that averaged $\geq 60 \mathrm{~min} /$ week of online yoga and those that averaged less. Randomized controlled trials that explore varying doses (i.e., different durations and frequencies) of yoga, or other forms of meditative movement, could determine the minimal dose at which effects of meditative movement begin to occur.

Research that identifies the effectiveness of meditative movement for hematological cancer patients is important in providing justification for implementing meditative movement into clinical practice as an evidence-based CIM strategy. Hematological cancer patients/survivors often suffer from long-term co-morbidities that ultimately end up affecting overall QoL [5-7]. The availability of evidence-based CIM strategies will be beneficial for cancer patients in the maintenance of their long-term health and QoL.

\section{Limitations}

Even though this review identified some preliminary evidence demonstrating the potential benefits of meditative movement for hematological cancer patients, there are limitations to note. First, the body of literature from which we draw our conclusions is very small. Our systematic review yielded only four studies. It is always possible that we missed some publications that could have been included in the review and could have changed our findings. We also only included articles published in English, which may have caused us to miss relevant articles published in other languages. Second, inherent with any systematic review is the possibility of publication and location bias $[28,29]$.

\section{Conclusions}

There is preliminary evidence that meditative movement may be beneficial for hematological cancer patient outcomes, particularly fatigue and sleep quality. There has been no research to date investigating the effects of tai chi on hematological cancer patients. Future research is needed to establish the effects of yoga, qigong, and tai chi on symptoms and QoL in hematological cancer patients. Future studies should also aim to determine the minimal dose at which effects begin to occur in hematological cancer patients.

\section{Authorship and Contributorship}

$\mathrm{RE}$ conceptualized the literature review, performed the literature review after search results had been obtained, interpreted the literature review results, conducted the quality assessment, drafted the manuscript, and edited/revised the manuscript; JH edited/revised the manuscript; AD edited/revised the manuscript; HK edited/revised the manuscript; LL edited/revised the manuscript; LM performed the literature search and conducted the quality assessment; RM edited/ revised the manuscript. All authors read and approved the final manuscript.

\section{Acknowledgements}

The authors do not report any acknowledgements.

\section{Funding}

The authors do not report any sources of funding.

\section{Competing Interests}

The authors do not report any competing interests.

\section{References}

1. http://www.lls.org/facts-and-statistics/facts-and-statistics-overview

2. https://www.1ls.org/treatment $\% 20 /$ types-of-treatment

3. https://www.leukaemia.org.au/disease-information/

4. Irwin M (2012) ACSM's guide to exercise and cancer survivorship. Human Kinetics Champaign, IL.

5. Bryant AL, Walton AL, Shaw-Kokot J, Mayer DK, Reeve BB (2015) Patient-reported symptoms and quality of life in adults with acute leukemia: a systematic review'. Oncol Nurs Forum 42: E91-E101.

6. Muffly LS, Hlubocky FJ, Khan N, Wroblewski K, Breitenbach K, et al. (2016) Psychological morbidities in adolescent and young adult blood cancer patients during curative-intent therapy and early survivorship. Cancer 122: 954-961.

7. Wang Z, Liu L, Shi M, Wang L (2016) Exploring correlations between positive psychological resources and symptoms of psychological distress among hematological cancer patients: a cross-sectional study. Psychol Health Med 21: 571-582.

8. Horneber M, Bueschel G, Dennert G, Less D, Ritter E, et al. (2012) 'How many cancer patients use complementary and alternative medicine: a systematic review and metaanalysis', Integr Cancer Ther 11: 187-203.

9. Fouladbakhsh JM, Stommel M (2008) Comparative analysis of CAM use in the US cancer and noncancer populations. Journal of Complementary and Integrative Medicine 5: 1.

10. Larkey L, Jahnke R, Etnier J, Gonzalez J (2009) Meditative movement as a category of exercise: implications for research. J Phys Act Health 6: 230-238.

11. Fouladbakhsh JM, Stommel M (2010) Gender, symptom experience, and use of complementary and alternative medicine practices among cancer survivors in the US cancer population. Oncol Nurs Forum 37: E7-E15.

12. Buffart LM, Van Uffelen JG, Riphagen II, Brug J, Van Mechelen W, et al. (2012) Physical and psychosocial benefits of yoga in cancer patients and survivors, a systematic review and meta-analysis of randomized controlled trials. BMC Cancer 12: 559.

13. Cote A, Daneault S (2012) Effect of yoga on patients with cancer: our current understanding. Can Fam Physician 58: e475-479.

14. Herder H, Parlour L, Jenkins V (2012) Randomised controlled trials of yoga interventions for women with breast cancer: a systematic literature review. Support Care Cancer 20: 3055-3064.

15. Sadja J, Mills PJ (2013) Effects of yoga interventions on fatigue in cancer patients and survivors: a systematic review of randomized controlled trials. Explore (NY) 9: 232-243. 
Eckert R (2018) The use of meditative movement as a non-pharmacologic approach in hematological cancer patients: A systematic review of the literature

16. Oh B, Butow P, Mullan B, Hale A, Lee MS, Guo X, et al. (2012) A critical review of the effects of medical Qigong on quality of life, immune function, and survival in cancer patients. Integr Cancer Ther 11: 101-110.

17. Zeng Y, Luo T, Xie H, Huang M, Cheng AS (2014b) Health benefits of qigong or tai chi for cancer patients: a systematic review and meta-analyses. Complement Ther Med 22: $173-186$.

18. Downs SH, Black N (1998) The feasibility of creating a checklist for the assessment of the methodological quality both of randomised and non-randomised studies of health care interventions. J Epidemiol Community Health 52: 377-384.

19. Hooper P, Jutai JW, Strong G, Russell-Minda E (2008) Age-related macular degeneration and low-vision rehabilitation: a systematic review. Can J Ophthalmol 43: 180-187.

20. Yeh M, Chung Y (2016) A randomized controlled trial of qigong on fatigue and sleep quality for non-Hodgkin's lymphoma patients undergoing chemotherapy. Eur J Oncol Nurs 23: 81-86.

21. Cohen L, Warneke C, Fouladi RT, Rodriguez M, Chaoul-Reich A (2004) Psychological adjustment and sleep quality in a randomized trial of the effects of a Tibetan yoga intervention in patients with lymphoma. Cancer 100: 2253-2260.

22. Chuang T, Yeh M, Chung Y (2017) A nurse facilitated mind-body interactive exercise (Chan-Chuang qigong) improves the health status of non-Hodgkin lymphoma patients receiving chemotherapy: randomised controlled trial. Int J Nurs Stud 69: 25-33.
23. Huberty J, Eckert R, Gowin K, Mitchell J, Dueck AC, et al. (2017) Feasibility study of online-streamed yoga for symptom management in patients with myeloproliferative neoplasms. Haematologica 102: e384-e388.

24. Rao MR, Raghuram N, Nagendra H, Gopinath K, Srinath B, et al. (2009) Anxiolytic effects of a yoga program in early breast cancer patients undergoing conventional treatment: a randomized controlled trial. Complement Ther Med 17: 1-8.

25. Raghavendra RM, Vadiraja H, Nagarathna R, Nagendra H, Rekha M, et al. (2009) Effects of a yoga program on cortisol rhythm and mood states in early breast cancer patients undergoing adjuvant radiotherapy: a randomized controlled trial. Integr Cancer Ther 8: 37-46.

26. Vadiraja SH, Rao MR, Nagendra RH, Nagarathna R, Rekha M, et al. (2009) Effects of yoga on symptom management in breast cancer patients: A randomized controlled trial. International Journal of Yoga 2: 73-79.

27. Zeng Y, Luo T, Xie H, Huang M, Cheng AS (2014a) Health benefits of qigong or tai chi for cancer patients: a systematic review and meta-analyses. Complement Ther Med 22: $173-186$.

28. Dickersin K (1990) The existence of publication bias and risk factors for its occurrence. JAMA 263: 1385-1389. [Crossref]

29. Ernst E, Pittler MH (1997) Alternative therapy bias. Nature 385: 480. [Crossref]

Copyright: @2018 Eckert R. This is an open-access article distributed under the terms of the Creative Commons Attribution License, which permits unrestricted use, distribution, and reproduction in any medium, provided the original author and source are credited. 